\title{
Ion migration in crystalline and amorphous $\mathrm{HfO}_{X}$
}

Marcel Schie, Michael P. Müller, Martin Salinga, Rainer Waser, and Roger A. De Souza

Citation: The Journal of Chemical Physics 146, 094508 (2017); doi: 10.1063/1.4977453

View online: http://dx.doi.org/10.1063/1.4977453

View Table of Contents: http://aip.scitation.org/toc/jcp/146/9

Published by the American Institute of Physics

\section{Articles you may be interested in}

Oxygen migration during resistance switching and failure of hafnium oxide memristors

The Journal of Chemical Physics 110, 103503103503 (2017); 10.1063/1.4974535

Atomic layer deposition and properties of mixed Ta2O5 and $\mathrm{ZrO} 2$ films

The Journal of Chemical Physics 7, 025001025001 (2017); 10.1063/1.4975928

Investigating the origins of high multilevel resistive switching in forming free $\mathrm{Ti} / \mathrm{TiO}-\mathrm{x}-$ based memory devices through experiments and simulations

The Journal of Chemical Physics 121, 094501094501 (2017); 10.1063/1.4977063

Enhancing ferroelectricity in dopant-free hafnium oxide

The Journal of Chemical Physics 110, 022903022903 (2017); 10.1063/1.4973928

Evidence for oxygen vacancies movement during wake-up in ferroelectric hafnium oxide

The Journal of Chemical Physics 108, 032903032903 (2016); 10.1063/1.4940370

The role of nitrogen doping in ALD Ta2O5 and its influence on multilevel cell switching in RRAM

The Journal of Chemical Physics 110, 102902102902 (2017); 10.1063/1.4978033

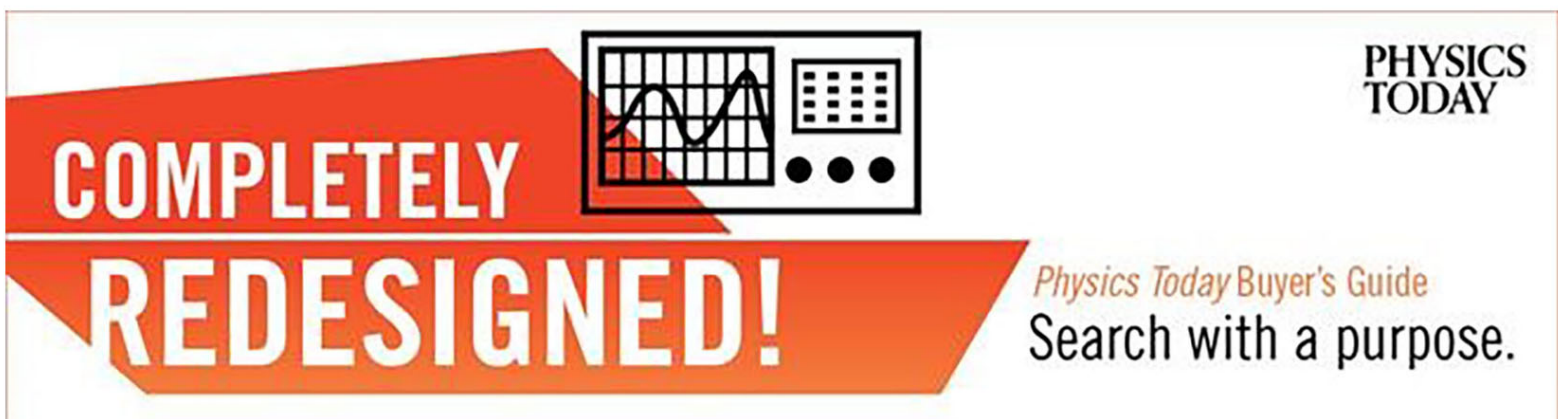




\title{
Ion migration in crystalline and amorphous $\mathrm{HfO}_{x}$
}

\author{
Marcel Schie, ${ }^{1, \text { a) }}$ Michael P. Müller, ${ }^{2}$ Martin Salinga, ${ }^{3}$ Rainer Waser, ${ }^{1}$ \\ and Roger A. De Souza ${ }^{2, b)}$ \\ ${ }^{1}$ Institute of Materials in Electrical Engineering and Information Technology II, RWTH Aachen University, \\ 52056 Aachen, Germany \\ ${ }^{2}$ Institute of Physical Chemistry, RWTH Aachen University and JARA-FIT, 52056 Aachen, Germany \\ ${ }^{3}$ I. Physikalisches Institut 1A, RWTH Aachen University, 52056 Aachen, Germany
}

(Received 6 December 2016; accepted 13 February 2017; published online 3 March 2017)

\begin{abstract}
The migration of ions in $\mathrm{HfO}_{x}$ was investigated by means of large-scale, classical molecular-dynamics simulations over the temperature range $1000 \leq T / \mathrm{K} \leq 2000$. Amorphous $\mathrm{HfO}_{x}$ was studied in both stoichiometric and oxygen-deficient forms (i.e., with $x=2$ and $x=1.9875$ ); oxygen-deficient cubic and monoclinic phases were also studied. The mean square displacement of oxygen ions was found to evolve linearly as a function of time for the crystalline phases, as expected, but displayed significant negative deviations from linear behavior for the amorphous phases, that is, the behavior was sub-diffusive. That oxygen-ion migration was observed for the stoichiometric amorphous phase argues strongly against applying the traditional model of vacancy-mediated migration in crystals to amorphous $\mathrm{HfO}_{2}$. In addition, cation migration, whilst not observed for the crystalline phases (as no cation defects were present), was observed for both amorphous phases. In order to obtain activation enthalpies of migration, the residence times of the migrating ions were analyzed. The analysis reveals four activation enthalpies for the two amorphous phases: $0.29 \mathrm{eV}, 0.46 \mathrm{eV}$, and $0.66 \mathrm{eV}$ (values very close to those obtained for the monoclinic structure) plus a higher enthalpy of at least $0.85 \mathrm{eV}$. In comparison, the cubic phase is characterized by a single value of $0.43 \mathrm{eV}$. Simple kinetic Monte Carlo simulations suggest that the sub-diffusive behavior arises from nanoscale confinement of the migrating ions. Published by AIP Publishing. [http://dx.doi.org/10.1063/1.4977453]
\end{abstract}

\section{INTRODUCTION}

Hafnia $\left(\mathrm{HfO}_{x}\right)$ has achieved unprecedented importance in modern information technologies, primarily because of its use as the gate dielectric in MOSFET devices but also, more recently, because of its emergence as a promising candidate for non-volatile resistive switching memories (ReRAMs). Both devices, though utilizing the same material, are based on opposing paradigms. In a MOSFET, the gate dielectric should be characterized by low point-defect densities and low ionic conductivity. A resistive switch, in contrast, requires high (local) defect densities and, in particular, operationspecific ionic conductivity: to switch the memory state, the (local) ionic conductivity should be high, but during readout and storage, it should be negligible. In a MOSFET or ReRAM device, $\mathrm{HfO}_{x}$ films can-depending on the processing procedures-be either crystalline or amorphous, and hence it is necessary to understand ion transport in both phases and to elucidate the similarities and differences in behavior. Ion transport, in contrast to electronic transport, ${ }^{1}$ in $\mathrm{HfO}_{x}$ has only been examined in a few publications, however (in crystalline phases $^{2-5}$ and in the amorphous state ${ }^{6-9}$ ), and no general picture of the behavior has as yet emerged.

\footnotetext{
Note: This paper is dedicated to Professor John A. Kilner on the occasion of his 70th birthday.

a) Electronic mail: schie@iwe.rwth-aachen.de

b) Electronic mail: desouza@pc.rwth-aachen.de
}

Atomistic simulations constitute a powerful method for examining ion transport because one has total control over the system combined with the ability to study processes directly on the atomistic scale. Clima et al. ${ }^{7-9}$ for example, analyzed the transport of oxygen ions in amorphous $\mathrm{HfO}_{x}$ in detail using $a b$ initio molecular-dynamics (MD) simulations. They used simulation cells that were rather small (containing only 96 ions); consequently, the periodic repetition of the cell, as implemented in the simulations, imposes a long-range order that is missing in an amorphous solid. Broglia et al. ${ }^{6}$ employed empirical pair-potentials (EPPs) to investigate oxygen diffusion in stoichiometric, amorphous $\mathrm{HfO}_{2}$ but obtained very high activation energies of oxygen diffusion of ca. $2 \mathrm{eV}$. It is unclear whether these high values are characteristic of the amorphous cells examined by Broglia et al. ${ }^{6}$ or are due to the EPP derived and used by Broglia et al.

In trying to understand diffusion in their amorphous simulation cells, Clima et $_{\text {al. }}{ }^{8}$ defined a defect similar to an oxygen vacancy as a void near an under-coordinated Hf cation; in contrast, Broglia et al. ${ }^{6}$ suggested that oxygen motion can be described in terms of an "interstitial" mechanism and that the introduction of oxygen vacancies would further promote the diffusion. We caution strongly against such interpretations, in which concepts from crystalline materials-point defectsare applied to amorphous materials. Defining point defects for an amorphous structure should generally be avoided as there is no ideal (reference) structure: for the crystalline state, the reference structure is the perfect lattice. One may think of 
defining defects based on under- or over-coordinated species, ${ }^{10}$ but as we will discuss later, this is problematic for amorphous hafnia. This problem is well-known to researchers in the glass community ${ }^{11-17}$ but seems to be ignored by those whose experience is mainly crystalline systems.

In this study, we address the topic of ionic transport in crystalline and amorphous $\mathrm{HfO}_{x}$ by performing classical molecular-dynamics simulations. We use large simulation supercells containing ca. 12000 ions and well-established pair potentials. ${ }^{18}$ In order to gain deeper understanding of ion migration in amorphous $\mathrm{HfO}_{x}$, we study the migration of $\mathrm{O}^{2-}$ anions and $\mathrm{Hf}^{4+}$ cations using three different methods. First, we monitor and evaluate the mean square displacement of both ion species from the MD simulations; then we estimate the activation barriers for migration based on the residence times of the migrating species, extending a method used by Lammert et al. ${ }^{14}$ and Helfferich et al. $;{ }^{19}$ and finally, we propose an explanation of sub-diffusive behavior in amorphous hafnia-nanoscale confinement-and we provide support for this model with simple kinetic Monte Carlo simulations.

\section{COMPUTATIONAL METHODOLOGY}

All static and dynamic molecular simulations conducted in this study are based on the Born model of polar solids. The constituent ions interact with each other through longrange coulomb interactions between ions that bear their formal charges, e.g., $\mathrm{O}^{2-}$ and $\mathrm{Hf}^{4+}$, and through short-range interactions implemented as parametrized Buckingham potentials of the form $\phi=A \cdot \exp ^{(-r / \rho)}-\frac{C}{r^{6}}$. In essence, the system is represented in terms of two-body, spherically symmetrical potentials. We used the set of empirical pair-potentials derived by Lewis and Catlow ${ }^{18}$ that have been shown over the last three decades to provide a good description of diverse ionic oxides; ${ }^{20-24}$ a list of the parameters is given in Table I. The cell vectors and ion positions for the crystalline phases were taken from the work of Jaffe $e t$ al. $^{25}$ and relaxed with the GULP ${ }^{26}$ code to the minimum cell energy.

Broglia et al. ${ }^{6}$ derived and employed Pedone-type ${ }^{27}$ EPP for $\mathrm{HfO}_{2}$, and although these EPP reproduce the lattice constants of hafnia (cubic, monoclinic) and of $\mathrm{HfSiO}_{4}$ well, they have not been shown to account for oxygen migration in crystalline phases. Indeed, static calculations with the GULP code (performed in this study) yield a very high migration enthalpy for oxygen-vacancy migration in cubic hafnia of $\Delta H_{\mathrm{m}}=1.67 \mathrm{eV}$. The EPP of Broglia ${ }^{6}$ are, therefore, not used for our simulations. We also conducted preliminary studies with the chemically more complex charge-optimised many-body (COMB) potentials. ${ }^{28}$ As shown in Fig. S1 in the

TABLE I. Parameters for the empirical Buckingham potential by Lewis and Catlow. ${ }^{18}$

\begin{tabular}{lcccc}
\hline \hline & $A(\mathrm{eV})$ & $\rho(\AA)$ & $C\left(\mathrm{eV} \cdot \AA^{6}\right)$ & $z(\mathrm{e})$ \\
\hline $\mathrm{H}^{4+}-\mathrm{O}^{2-}$ & 1454.6 & 0.3500 & 0.000 & +4.0 \\
$\mathrm{O}^{2-}-\mathrm{O}^{2-}$ & 22764.3 & 0.1490 & 27.879 & -2.0 \\
$\mathrm{Y}^{3+}-\mathrm{O}^{2-}$ & 1345.1 & 0.3491 & 0.000 & +3.0 \\
\hline \hline
\end{tabular}

supplementary material, the activation enthalpy of oxygen migration is remarkably low, and therefore these potentials were not used either. As will be shown below, the comparatively simple Lewis-Catlow EPP ${ }^{18}$ provide physically reasonable behavior for the crystalline phases, and in particular for ion migration.

The molecular-dynamics (MD) simulations employed the velocity Verlet algorithm ${ }^{29}$ to propagate the system through phase space and the thermostat and barostat by Martyna et al. ${ }^{30}$ to establish a $N p T$ ensemble (constant particle number $N$, pressure $p$, and temperature $T$ ). The DL_POLY $\operatorname{code}^{31}$ was used for all MD simulations. A $10 a \times 10 a \times 10 a$ supercell $(=12000$ ions) was generated, and 50 oxygen ions were removed from randomly chosen lattice sites. The surplus charge was compensated by a homogeneous background charge (this has been shown for oxygen-vacancy dynamics in $\mathrm{SrTiO}_{3}{ }^{32}$ to be a physically reasonable procedure). We simulated both cubic and monoclinic supercells, as well as a stoichiometric and an oxygen-deficient amorphous cell. The amorphous exemplars were generated by a melt and quench procedure from the cubic cell. That is, the supercell was first heated to a temperature of $10^{4} \mathrm{~K}$ within $100 \mathrm{ps}$, held at this temperature for further $900 \mathrm{ps}$, and then quenched to $1000 \mathrm{~K}$ within $100 \mathrm{ps}$ and again held for further $900 \mathrm{ps}$.

For the MD simulations of ion migration, each system was first equilibrated for $100 \mathrm{ps}$, at which point the cell's energy and volume were found to be constant; all structures (cubic, monoclinic, and amorphous) remained stable according to their respective radial distribution functions (RDFs) for temperatures up to $2000 \mathrm{~K}$. Ion migration was examined in $1 \mathrm{~ns}$ long MD runs, in which the specific mean square displacements $\operatorname{MSD}(t)$ for $\mathrm{Hf}^{4+}$ and $\mathrm{O}^{2-}$ as well as all ion positions were saved every picosecond for later analysis.

\section{PROPERTIES OF CRYSTALLINE $\mathrm{HfO}_{2}$}

\section{A. Thermodynamic properties}

Static simulations were employed to examine the energy of a cell as a function of its volume. Data obtained for the cubic, orthorhombic (cotunnite), monoclinic, and tetragonal phases of $\mathrm{HfO}_{2}$, as well as for the amorphous phase that we prepared, are shown in Fig. 1. The data, it is emphasised, refer to room temperature, that is, to the temperature of the lattice to which the EPP were fitted. The cubic phase is thus seen to have the lowest energy minimum, although the monoclinic phase is the most stable at ambient temperature and pressure $;{ }^{25}$ the orthorhombic and monoclinic minima are slightly higher and the tetragonal minima clearly higher in energy. This preference for the cubic phase is tentatively ascribed to the fact that spherical pair-potentials tend to prefer a high-symmetry (cubic) over lower-symmetry structures. Compared with the initial cell parameters from the work of Jaffe et al. ${ }^{25}$ the relaxed tetragonal structure exhibits one distinctly larger cell parameter $(\Delta c / c \approx 25 \%)$ and a different position for the oxygen ion; the other phases remained close to their respective starting structures. The heat capacity at constant pressure and thermal expansion are also described well by these EPP (see Fig. S2 in the supplementary material). 


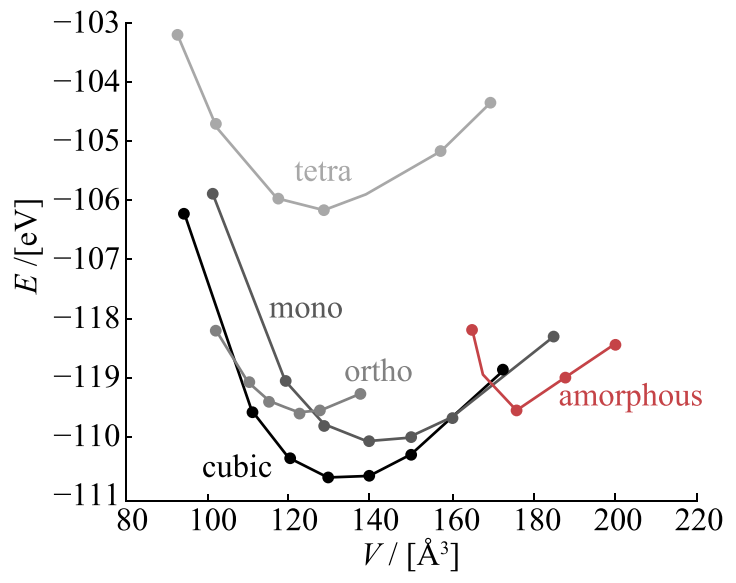

FIG. 1. Energy vs. volume diagram for the various phases of $\mathrm{HfO}_{2}$. The cubic phase has the lowest energy minimum and thus is the preferred structure for the used set of empirical pair-potentials.

\section{B. Diffusion in $\mathrm{HfO}_{2}$}

In order to study the diffusion of oxygen by a vacancy mechanism in cubic and monoclinic $\mathrm{HfO}_{1.9875}$, we obtained from the trajectories $r_{i}(t)$ of all $N$ oxygen ions their mean square displacement as a function of time

$$
\operatorname{MSD}_{\mathrm{O}}(t)=\frac{1}{N} \cdot \sum_{i=1}^{N}\left[r_{i}\left(t_{0}\right)-r_{i}\left(t_{0}+t\right)\right]^{2}
$$

Data are shown for the cubic phase at various temperatures in Fig. 2. Describing this behavior with the standard Einstein relation,

$$
\operatorname{MSD}_{\mathrm{O}}(t)=6 D_{\mathrm{O}}^{\star} t+B,
$$

yields the tracer diffusion coefficient of oxygen, $D_{\mathrm{O}}^{\star}$. $B$ describes the thermal vibration of the ions around their equilibrium lattice sites. The same analysis was conducted for the $\mathrm{Hf}$ species. The linear increase of $\mathrm{MSD}_{\mathrm{O}}$ with time indicates that the oxygen ions are mobile. In contrast, the constant $\mathrm{MSD}_{\mathrm{Hf}}$ indicates that the hafnium ions are immobile: this immobility is due to the lack of cation defects (neither vacancies nor interstitials were introduced into the simulation cells, and the generation of Frenkel pairs is extremely unlikely, given that predicted Frenkel energies in fluorite oxides are $10 \mathrm{eV}$ or higher ${ }^{33,34}$ ); and it is due to the high activation barriers for cation migration in fluorite oxides. ${ }^{35,36}$

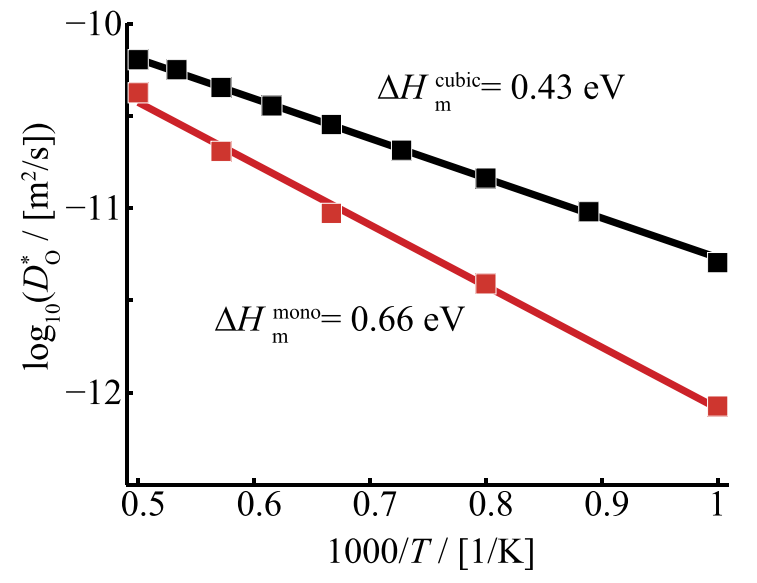

FIG. 3. Oxygen tracer diffusion coefficients $D_{\mathrm{O}}^{*}$ for cubic and monoclinic $\mathrm{HfO}_{1.9875}$ obtained by MD simulations as a function of inverse temperature.

Fig. 3 shows the oxygen tracer diffusion coefficients obtained for the two crystalline phases. Oxygen diffuses faster, and with a lower activation enthalpy of diffusion, in the structure with the higher symmetry; this result confirms the widely held belief ${ }^{37-39}$ that high-symmetry structures are preferred for fast ion transport. The activation enthalpy of oxygen diffusion is calculated to be $\Delta H_{\mathrm{mig}, \mathrm{O}}^{\text {cubic }}=0.43 \mathrm{eV}$ for the cubic and $\Delta H_{\mathrm{mig}, \mathrm{O}}^{\mathrm{mono}}=0.66 \mathrm{eV}$ for the monoclinic structure. These results agree well with the activation enthalpies, $\Delta H_{\mathrm{mig}, \mathrm{O}}$ $=0.40-0.60 \mathrm{eV}$, extracted by Zafar et al. ${ }^{40}$ from currentvoltage studies of ultra-thin $\mathrm{HfO}_{x}$ films. There is also good agreement with the density functional theory (DFT) calculations by Capron et al. $^{3}$ of oxygen-vacancy migration in the monoclinic phase. They determined individual activation barriers of migration and predict for long-range diffusion an overall activation enthalpy of $\Delta H_{\mathrm{mig}, \mathrm{O}}^{\mathrm{mono}}=0.69 \mathrm{eV}$ (with other individual barriers along this path of $\Delta H_{\mathrm{mig}, \mathrm{O}}^{\mathrm{mono}}=0.23 \mathrm{eV}$ and $\Delta H_{\text {mig, } \mathrm{O}}^{\mathrm{mono}}=0.49 \mathrm{eV}$ ). The activation enthalpy of diffusion we

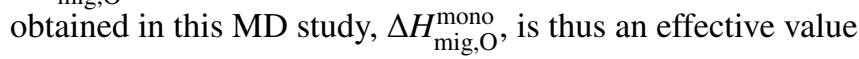
dominated by the highest barrier along the lowest-energy path.

Two other results are also mentioned at this point. First, the directional $D^{*}$ values along the $a, b$, and $c$ axes of the monoclinic cell are different, with $D_{a}^{*}: D_{b, c}^{*} \approx 2: 1$, as expected for an anisotropic crystal, see Fig. S3. Second, the oxygen tracer diffusivity was examined as a function of yttrium dopant concentration. As shown in Fig. S4 of the supplementary material, a maximum was observed at ca. $10 \%$, as seen in experiment. ${ }^{41,42}$ Thus, combining these two results with the results of Fig. 3,
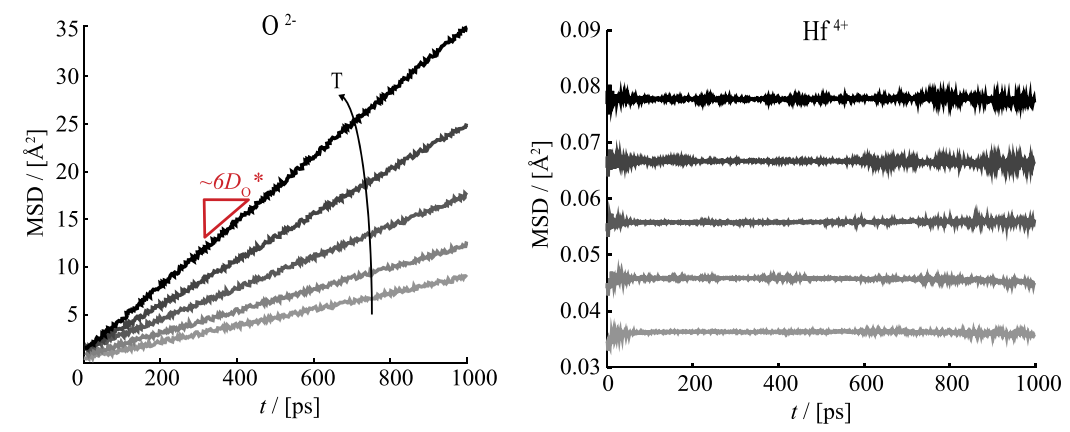

FIG. 2. Mean square displacement (MSD) for $\mathrm{O}^{2-}$ anions (left) and $\mathrm{Hf}^{4+}$ cations (right) in cubic $\mathrm{HfO}_{1.9875}$ for $1000 \leq T / \mathrm{K} \leq 2000$ (every $250 \mathrm{~K}$ ). The constant MSD values for hafnium indicate that no hafnium diffusion occurred (as no cation defects were present). 
we conclude that these simple EPP are able to describe oxygen-vacancy dynamics in crystalline $\mathrm{HfO}_{2}$ very well.

\section{MOLECULAR DYNAMICS OF AMORPHOUS $\mathrm{HfO}_{x}$}

\section{A. RDFs and the glassy phase}

In Fig. 4, we compare the RDFs at $T=1000 \mathrm{~K}$ calculated for the stoichiometric amorphous cell with those for the stoichiometric cubic and monoclinic cells (the oxygen deficient cells only differ imperceptibly). The amorphous supercell exhibits pronounced first-nearest-neighbor peaks, small and broadened second-nearest-neighbor peaks, and no long-range order beyond $8 \AA$. In this way, the RDFs indicate that we have indeed prepared an amorphous phase. Comparisons with the RDFs from the classical MD simulations by Broglia et al. ${ }^{6}$ and with that from the $a b$ initio MD calculations by Clima et ll $^{7}$ (but only below $5 \AA$ because of the small supercell size they used) show good agreement. We also emphasize that the RDFs of the amorphous cells were unchanged after all MD (diffusion) simulations.

The coordination numbers of $\mathrm{Hf}$ and $\mathrm{O}$ were also extracted from the simulations. In the monoclinic structure, Hf exhibits seven-fold coordination, while for $\mathrm{O}$, planes of three-fold and four-fold coordinations alternate (ratio of 1:1). ${ }^{33} \mathrm{In}$ am- $\mathrm{HfO}_{x}$ (see Fig. 5), Hf is mainly found in six- and seven-fold coordination polyhedra, with some five-, eight-, and nine-fold coordination polyhedra also evident; for oxygen, the ratio of three- to four-fold coordination changes to almost 2:1 and two- and five-fold coordinated species are also found. The important fact here is that both distributions are relatively broad. If $\mathrm{Hf}$ in $\mathrm{am}-\mathrm{HfO}_{x}$ were to be situated overwhelmingly in seven-fold coordination polyhedra, with only a few six- and eight-fold coordination polyhedra present, one could term the deviations from seven-fold coordination as vacancy (six-fold) and interstitial (eight-fold) defects, as has been reported for $\mathrm{SiO}_{x} \cdot{ }^{10}$ This is not the case, however, for

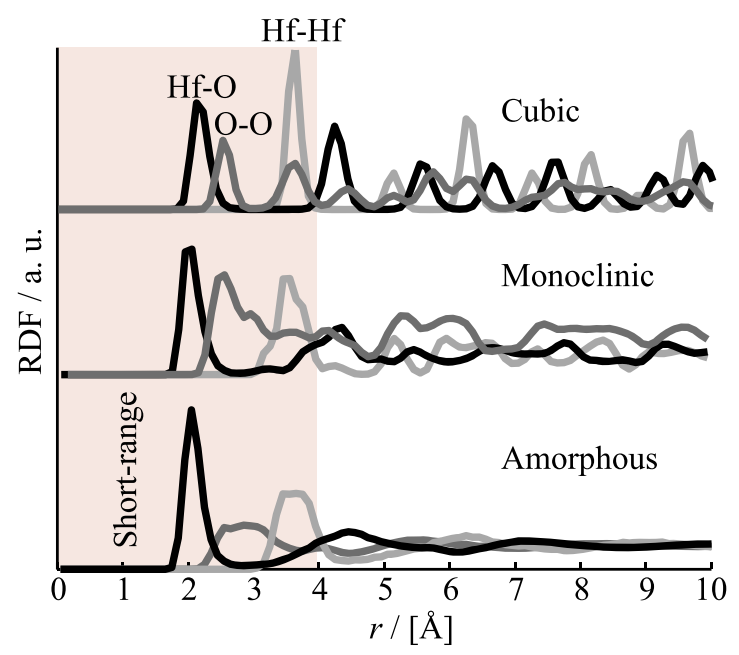

FIG. 4. Radial distribution functions for $\mathrm{Hf}-\mathrm{O}$ (black), O-O (dark grey), and $\mathrm{Hf}-\mathrm{Hf}$ (light grey) distances in $\mathrm{HfO}_{2}$ at $T=1000 \mathrm{~K}$. The lower graph refers to the amorphous supercell without defects (the results for the amorphous cell with defects are identical).

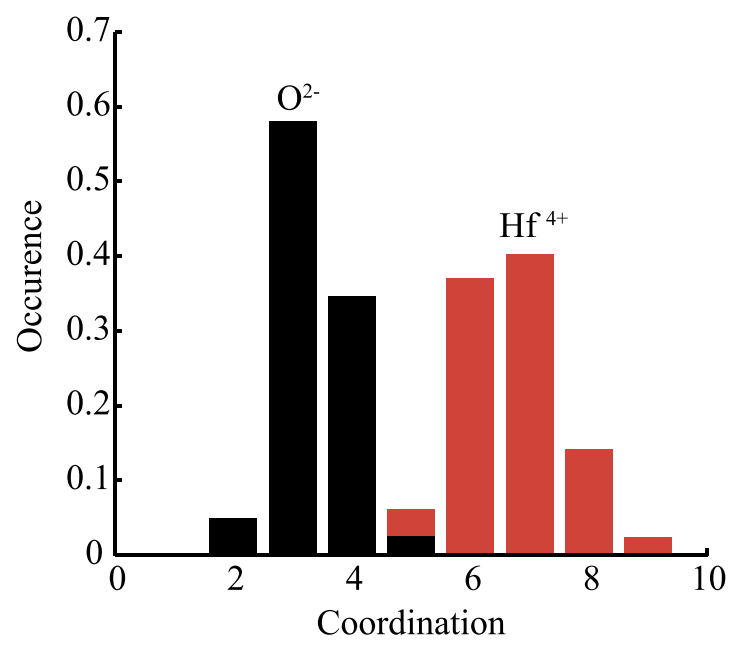

FIG. 5. Coordination numbers for oxygen-deficient amorphous $\mathrm{HfO}_{x}$ at $T$ $=1000 \mathrm{~K}$.

am- $\mathrm{HfO}_{x}$ according to our simulations and those of Clima et al. ${ }^{7}$ and Ceresoli and Vanderbilt. ${ }^{43}$ For this reason, we argue strongly against using the language of crystal defects to interpret diffusion in this amorphous system. A further argument arises from the sites that oxygen ions migrate to and from (see later).

In order to examine the stability of the amorphous phases and to ensure that no structural relaxations are present, as mentioned by Angell et al. ${ }^{44}$ we subjected the simulation cells to a quasi-static thermal cycle, in which the system was equilibrated at a range of (first increasing, then decreasing) temperatures. In Fig. 6, we plot the volume of the cell as a function of temperature during this heating and cooling cycle. Three different regimes can be identified: at the highest temperatures (above $5000 \mathrm{~K}$ ) one has the molten phase; at the lowest temperatures (below $2500 \mathrm{~K}$ ) one has the glassy state; and in between, where there is a bending in the behavior around $T=3500 \mathrm{~K}$, the structure may relax during experiments or simulations. The temperature range of our MD (diffusion) simulations lies within the glassy regime (below $2500 \mathrm{~K}$ ). Here negligible structural relaxations occur so that all ionic movements can be attributed to vibrations or diffusion.

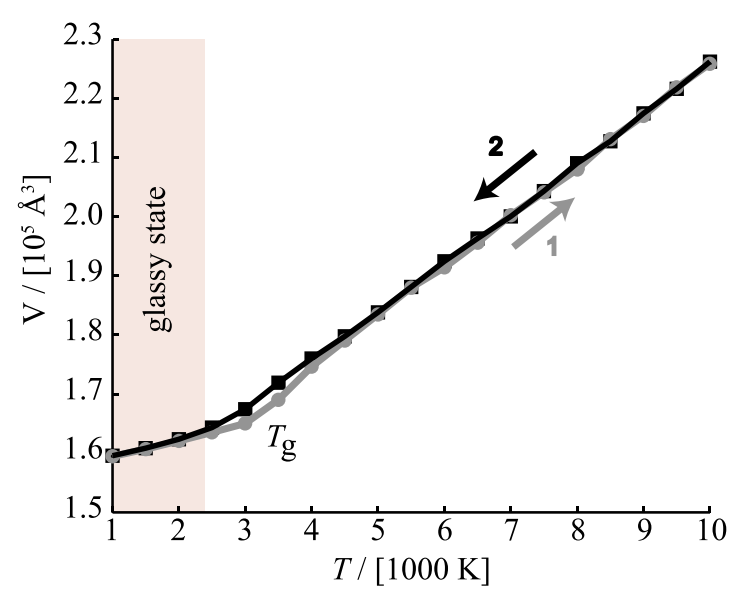

FIG. 6. Volume of amorphous $\mathrm{HfO}_{x}$ during a molecular dynamics heating (grey) and cooling (black) cycle started from the amorphous phase. 


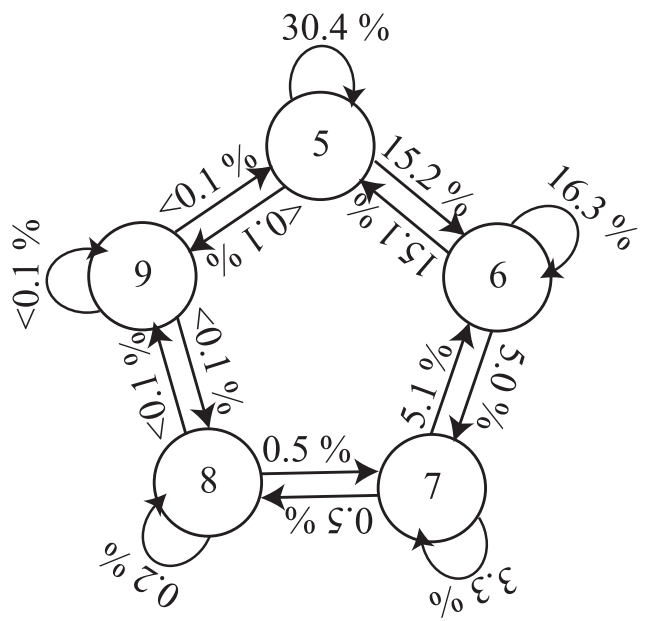

FIG. 7. Statistics showing the relative number of oxygen ion jumps from and to different coordinated environments at $T=1000 \mathrm{~K}$.

\section{B. Diffusion}

We first examined the coordination statistics of the ion jumps. Let us hypothesize that the standard defect picture is an appropriate description of ion migration in am- $\mathrm{HfO}_{x}$. In this case, one would expect to observe only ion jumps from higher to lower coordinated environments: oxygen-ion jumps from eight-fold to seven-fold coordination polyhedra, for example, would correspond to interstitial migration, whereas oxygenion jumps from six-fold to five-fold coordination polyhedra would correspond to oxygen vacancies moving in the other direction. Thus, although a reference structure would be lacking, one could regard the migrating species as coordination defects (as has been argued for $\mathrm{SiO}_{x}{ }^{10}$ ). In the MD simulations, however, a comparable number of contrary ion jumps were detected (see Fig. 7). That is, oxygen ions were observed jumping from lower to higher coordination polyhedra (for instance, from seven-fold to eight-fold and in this way, producing sixfold and nine-fold coordination polyhedra). Such a behavior is clearly not describable in terms of the migration of point defects. It constitutes, therefore, a second argument against a point-defect description of am- $\mathrm{HfO}_{x}$.

The mean square displacements for $\mathrm{O}^{2-}$ as well as $\mathrm{Hf}^{4+}$ were calculated according to Eq. (1) and are shown in Fig. 8. Three major features are evident. First, both the oxygendeficient and the stoichiometric amorphous cells exhibit oxygen ion mobility. Second, significant Hf diffusion is also seen,

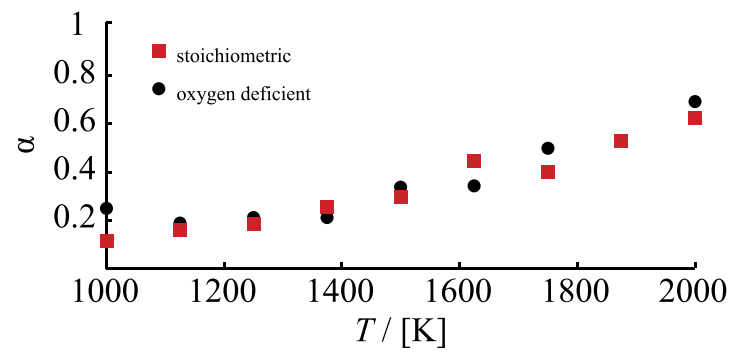

FIG. 9. Variation of $\alpha$ as a function of temperature $T$ for amorphous hafnia. $\alpha=1$ corresponds to diffusive behavior, $\alpha<1$ to sub-diffusive.

even though the cell is stoichiometric; these cations were found to be immobile in the crystalline phases (see Fig. 8). Third, both anion and cation diffusion occur sub-diffusively, following the proportionality

$$
\operatorname{MSD}(t) \propto t^{\alpha}
$$

with values of $\alpha$ obtained from the simulations (see Fig. 9) increasing with increasing temperature but remaining below unity at all temperatures and for the time scales examined. Obviously, modelling vacancy motion in am- $\mathrm{HfO}_{x}$ over short time- and length-scales in ReRAM devices will be complex, since $\alpha=f(t, T)$.

Determining diffusion coefficients from these data with Eq. (2) clearly makes no sense, since the behavior of the MSDs is not linear. One can define a generalized diffusivity, with units of $\left[\mathrm{m}^{2} \mathrm{~s}^{-\alpha}\right]$, but it is unclear how to compare such data with the true tracer diffusion coefficients obtained with Eq. (2) for the crystalline phases. Furthermore, there is no physical basis for extracting an activation enthalpy of diffusion from the temperature dependence of the generalized diffusivity. It is noted, though, that after $t=1 \mathrm{~ns}$ at $2000 \mathrm{~K}$ the MSD of oxygen ions in the amorphous phase reaches the same value as in the cubic phase $\left(\mathrm{MSD}_{\mathrm{O}}^{\mathrm{am}} \approx \mathrm{MSD}_{\mathrm{O}}^{\text {cubic }}\right.$ ), while for lower temperatures the amorphous phase shows a distinctly smaller MSD. At all temperatures, $\mathrm{MSD}_{\mathrm{O}}^{\mathrm{am}}$ is approximately 6 times $\mathrm{MSD}_{\mathrm{Hf}}^{\mathrm{am}}$. In contrast to the behavior found for the monoclinic cell, the $\mathrm{MSD}_{\mathrm{O}}^{\mathrm{am}}$ values along the $x, y$, and $z$ directions of the amorphous cell showed no anisotropy, with $\mathrm{MSD}_{x}: \mathrm{MSD}_{y}: \mathrm{MSD}_{z} \approx 1: 1: 1$ (see Fig. S5 of the supplementary material). That is, the amorphous cells behaved as expected.

In order to estimate the activation barriers for ion migration in am- $\mathrm{HfO}_{x}$, we take an alternative approach. Namely,
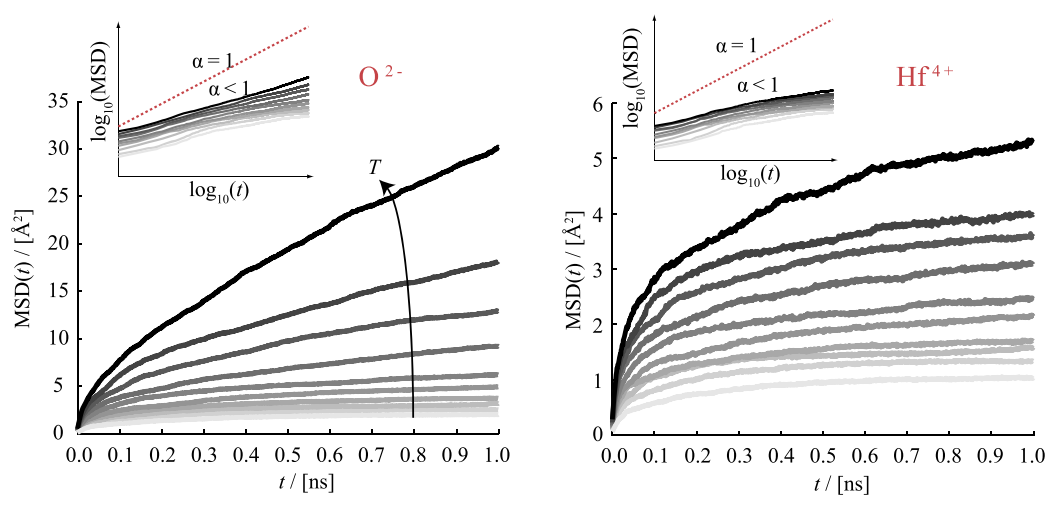

FIG. 8. MSD of oxygen and hafnium in the stoichiometric amorphous $\mathrm{HfO}_{2}$ cell for $875 \leq T / \mathrm{K} \leq 2000$ (every $125 \mathrm{~K})$. The bending in the MSD and the $\alpha<1$ in the $\log$-log plot depict the sub-diffusive behavior. 
we examine at a given temperature the distribution of residence times of the migrating species, extending the (waiting time) approach of Lammert et al..$^{14}$ and Helfferich et al. ${ }^{19}$ The residence time $\tau$ is defined here as the time an ion remains within a small volume around its initial position, even though it could migrate to its final position. This residence time is different from the rest time, which is the period of time for which an ion remains within a small volume around its initial position, but has no opportunity to migrate because its final position is occupied. Residence and waiting times are distinguished from each other in the following manner: Once an ion had jumped (allowing us to identify the final position), we determined how long beforehand this final position had been unoccupied. Thus, having determined the total time between two jumps of an ion-a jump was considered to have taken place if the ion's displacement was larger than $1.75 \AA$ (see Fig. 4) -we partitioned this total waiting time into a residence time and a rest time. The residence time distributions obtained from the simulations (with the data being sorted into bins of width $1 \mathrm{ps}$ ) for the (oxygen deficient) cubic, monoclinic, and amorphous phases are shown in Fig. 10.

We assume that ion hopping occurs independently and stochastically, and hence that it can be considered as a Poisson process. Consequently, the probability that an ion has jumped between $t=0$ and $t=\tau$ is given by the cumulative distribution function

$$
F(\tau)=1-\mathrm{e}^{-\tau / \bar{\tau}},
$$

where $\bar{\tau}$ is the mean residence time of the ion. To analyse the data in Fig. 10, we require the probability that an ion has jumped between $t=\tau-\Delta \tau$ and $t=\tau+\Delta \tau$ (with $\Delta \tau=1 / 2 \mathrm{ps}$ ), as this quantity is equal to the number of ions that executed jumps in this time period, $n_{\text {jumps }}$, relative to the total number of jumps observed over the simulation, $n_{\text {jumps }}^{\text {tot }}$, $\frac{n_{\text {jumps }}}{n_{\text {jumps }}^{\text {tot }}}=F(\tau+\Delta \tau)-F(\tau-\Delta \tau)=\mathrm{e}^{-\tau / \bar{\tau}}\left[\mathrm{e}^{\Delta \tau / \bar{\tau}}-\mathrm{e}^{-\Delta \tau / \bar{\tau}}\right]$.

If there is more than one type of barrier present in the system, each barrier has its own mean residence time, and the distribution of residence times is given by

$$
\frac{n_{\text {jumps }}}{n_{\text {jumps }}^{\text {tot }}}=\sum_{j} k_{j} \mathrm{e}^{-\tau / \bar{\tau}_{j}}\left[\mathrm{e}^{\Delta \tau / \overline{\tau_{j}}}-\mathrm{e}^{-\Delta \tau / \bar{\tau}_{j}}\right]
$$

with $k_{j}$ representing the relative amount of barriers overcome. The results of fitting Eq. (6) to the data to obtain $\bar{\tau}_{j}$ (and $k_{j}$ ) are shown as red lines in Fig. 10. Only in the case of the cubic system (one single barrier), it is stressed, is it clear a priori how many different barriers were probed by the migrating ions in the duration of the MD simulations. We discuss this issue below.

The last part of the analysis consists, first, of relating the mean residence times $\bar{\tau}_{j}$ to the jump frequencies $\gamma_{j}$ according to $\gamma_{j}=1 / \bar{\tau}_{j},{ }^{45}$ and second, of expressing $\gamma_{j}$ in terms of the activation enthalpy of migration, $\Delta H_{\mathrm{mig}, \mathrm{O}, j}$, through

$$
\gamma_{j}=\gamma_{0} \mathrm{e}^{-\frac{\Delta H_{\mathrm{mig}, \mathrm{O}, j}}{k_{\mathrm{B}} T}} .
$$

The temperature $T$ is the temperature of the MD simulation, and the attempt frequency was taken to be $\gamma_{0}=1.5 \cdot 10^{13} \mathrm{~s}^{-1}$ in order to reproduce the activation enthalpy of oxygen diffusion in cubic hafnia of $\Delta H_{\mathrm{mig}, \mathrm{O}}^{\text {cubic }}=0.43 \mathrm{eV}$ (see Fig. 2). This value of $\gamma_{0}$ is consistent with a migration entropy of a few $k_{\mathrm{B}}$ and a characteristic frequency $v_{0}$ calculated from the Debye temperature $\Theta_{\mathrm{D}}=483-549 \mathrm{~K}^{46,47}$ according to $v_{0}=\frac{k_{\mathrm{B}} \Theta_{\mathrm{D}}}{h}$. As a zero-order approximation, $\gamma_{0}$ is assumed to be constant for all processes; this, of course, neglects the fact that different structures, or even different jumps within a given structure, may have slightly different attempt frequencies.
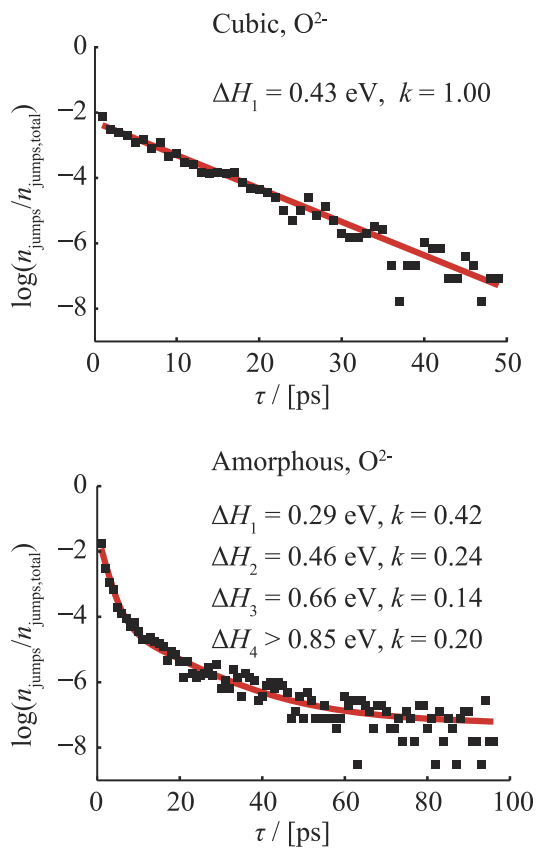
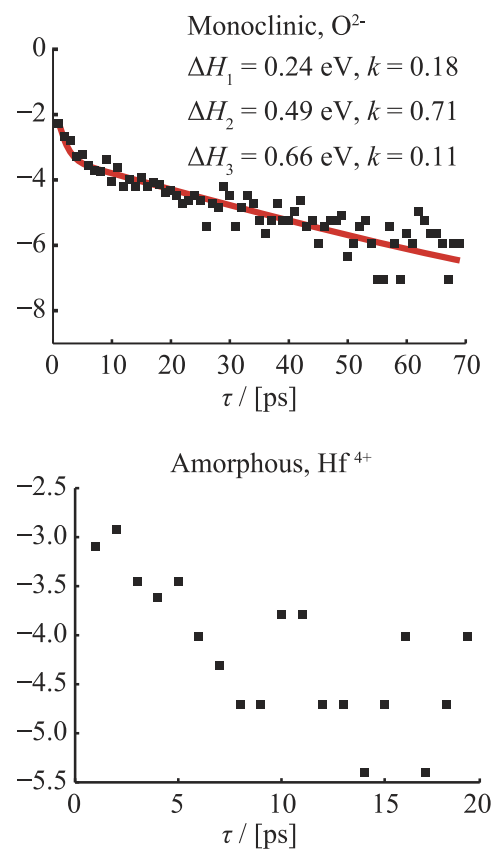

FIG. 10. Residence-time distributions at $T=1000 \mathrm{~K}$ for a cubic, a monoclinic, and an amorphous supercell (only the stoichiometric case is depicted; the defective case differs only imperceptibly in appearance and numbers). 
For the monoclinic cell, the analysis yielded three activation barriers, $\Delta H_{\text {mig, }, 11}^{\text {mono }}=0.24 \mathrm{eV}, \Delta H_{\text {mig, }, 2,2}^{\text {mono }}=0.49 \mathrm{eV}$, and $\Delta H_{\mathrm{mig}, \mathrm{O}, 3}^{\mathrm{mono}}=0.66 \mathrm{eV}$ (the errors in the energy barriers are at most $0.03 \mathrm{eV}$ and refer to the uncertainty of the fit and variations of $\gamma_{0}$ ). These values agree well with our previous result for oxygen tracer diffusion (see Fig. 3), as the highest barrier (along the lowest energy path) governs the behavior. Furthermore, all three barriers are consistent with the range of barriers determined by Capron et al. ${ }^{3}$ with DFT calculations. We were unable to describe the residence time distribution using only two barriers. In addition, we attempted to fit the data with four or more $\gamma_{j}$ contributions because Capron et al. predicted that higher energy barriers are present, albeit for other migration paths through the monoclinic crystal. The analysis did not reveal any additional barriers, however. The inclusion of a fourth barrier, for example, resulted in $\Delta H_{\mathrm{mig}, \mathrm{O}, 3}^{\mathrm{mono}}=\Delta H_{\mathrm{mig}, \mathrm{O}, 4}^{\mathrm{mono}}=0.66 \mathrm{eV}$, with equal $k_{j}$ s. Such high energy barriers were evidently not probed, therefore, by migrating ions in sufficient numbers during our MD simulations.

The analysis of the amorphous cells revealed four different barriers for oxygen migration: $\Delta H_{\mathrm{mig}, \mathrm{O}, 1}^{\mathrm{am}}=0.29 \mathrm{eV}$, $\Delta H_{\text {mig,O,2 }}^{\mathrm{am}}=0.46 \mathrm{eV}, \Delta H_{\mathrm{mig}, \mathrm{O}, 3}^{\mathrm{am}}=0.66 \mathrm{eV}$, and $\Delta H_{\mathrm{mig}, \mathrm{O}, 4}^{\mathrm{am}}$ $>0.85 \mathrm{eV}$ (with poor statistics for the latter preventing a more precise analysis). Higher energy barriers were not detected in this analysis. These values, it is emphasised, are identical for both oxygen-deficient and stoichiometric cells; more importantly, the three lowest values are very close to those obtained for the monoclinic cell. It is unclear which of these barriers will govern sub-diffusion, since we have no activation enthalpy for the overall process. Furthermore, the spatial distribution of the individual barriers will play a role in determining which barrier(s) is important, and this spatial distribution is unknown (and despite attempts to do so, it was not possible to extract this information from the simulations). For hafnium ions, an activation barrier of $\Delta H_{\text {mig,Hf, } 1}^{\mathrm{am}} \approx 0.3 \mathrm{eV}$ was determined, based on the analysis of the first $8 \mathrm{ps}$; poor statistics prevent, again, a more precise analysis and also the determination of further, higher energy barriers.
The close coincidence of values obtained for the amorphous and monoclinic structures is understandable because the near order in both phases (see Fig. 4) is similar, ${ }^{48}$ and it is this environment that is expected to govern the energetics of the migration process. Indeed, according to Genreith-Schriever et al. ${ }^{49}$ the repulsive Coulomb interactions between the migrating anion and the surrounding nextnearest-neighbour oxygen ions determine the energetics of anion migration in fluorite-based oxides.

There are two important points to make regarding the values of $\Delta H_{\text {mig }}$ obtained. First, they do not vary with temperature. That is, analysis of MD data for other temperatures yielded identical values of $\Delta H_{\mathrm{mig}}$. Second, the description in terms of discrete barriers is not necessarily unique. Traditional wisdom maintains that amorphous structures are characterized by a broad range of barriers ${ }^{50}$ whereas the residence time distribution for the amorphous phases could be described very well by 4 discrete barriers. We ascertained (not shown) that it is also possible to describe the data for the amorphous phases with 4 Gaussian-type distributions centered on the discrete values, but not with a continuum of values $\left(k_{j}=\right.$ const $\left.\forall \Delta H_{\text {mig }}\right)$. Interestingly, it is also possible to describe the residence time distribution for the monoclinic phase with 3 Gaussiantype distributions centered on the respective discrete values. Hence, if we consider the amorphous phases to be based on the monoclinic phase, a description with discrete barriers is preferable.

One physical model that yields sub-diffusive behavior is the sub-diffusive continuous time random walk as described by Metzler et al. ${ }^{51}$ Accordingly, we consider amorphous $\mathrm{HfO}_{x}$ to comprise small regions of high mobility (i.e., characterized by low activation barriers) that are separated from one another by much higher activation barriers. The mobile species are confined, therefore, by the high barriers for short times, and the behavior is subdiffusive. For long times, the species will also overcome the higher barriers and there is a transition to normal diffusive behavior. If the high-mobility regions are sufficiently large, an additional normal diffusion regime can be present at very short times. The model is illustrated schematically in Fig. 11(a). a)

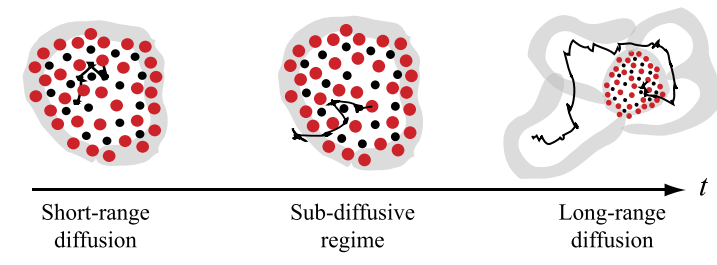

b)

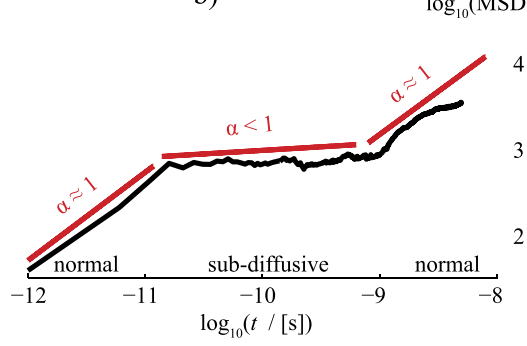

c)

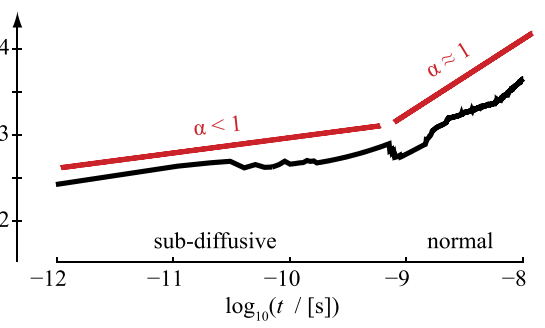

FIG. 11. Model of the diffusion behavior in amorphous $\mathrm{HfO}_{x}$ (a). The material is assumed to consist of regions of high ionic mobility separated by higher migration barriers. Results of simple kinetic Monte Carlo simulations with "larger" (b) and "smaller" (c) areas of high ionic mobility are shown for comparison. For the former, the diffusion for short times behaves normally while for the latter a normal diffusion regime for short times is not present. 
To test whether such a general model is consistent with our data, we performed simple kinetic Monte Carlo simulations. Specifically, the motion of independent random walkers in a two-dimensional energy landscapes was simulated. Three types of landscape were examined: (i) a statistically random (isotropic on average) distribution of energy barriers, (ii) small regions of high mobility separated by high energy barriers that confine motion, and (iii) large regions of high mobility separated by high energy barriers that confine motion. The high mobility areas are represented by the barriers calculated from the residence time analysis, and jump distances were taken from the RDF distributions (see Fig. 4). For the first type of energy landscapes, the simulations (not shown) yielded normal diffusive behavior for the time scales examined. The latter two energy landscapes, however, produced sub-diffusive behaviour, as shown in Figs. 11(b) and 11(c), only if confining barriers with energies higher than $1 \mathrm{eV}$ were introduced. For these simple, 2D simulations with static energy landscapes and high energy barriers of ca. $1 \mathrm{eV}$, a sub-diffusive behaviour was generally observed between $10 \mathrm{ps}$ and $1 \mathrm{~ns}$; for longer times, the behaviour became diffusive, as expected. These results should be considered primarily as confirmation of the simple model illustrated in Fig. 11 and not as confirmation of the MD results themselves. Thus we find that classical nanoscale confinement of migrating ions by a static energy landscape is sufficient to generate sub-diffusive behaviour. Further kMC simulations of three-dimensional (static and dynamic) energy landscapes with various distributions of energy barriers and further MD simulations are, however, required for deeper insights into sub-diffusion and the temporal transitions to normal diffusion.

\section{CONCLUSIONS}

We performed classical molecular dynamics simulations to study ion migration in amorphous and crystalline hafnia. The use of long simulation runs and large simulation cells revealed a number of differences in behavior.

- Ion migration in amorphous hafnia is evidently subdiffusive on the nanosecond time scale. There is no linear relationship between the distance migrated and the time allowed for migration. Nanoscale confinement is held to be responsible for this effect.

- Hafnium diffusion was observed in amorphous cells, whereas no diffusion of hafnium was detected in crystalline cells of identical composition.

- The use of point-defect descriptions is to be avoided for ion migration in amorphous hafnia because the definition of defects and mobile species is not possible.

\section{SUPPLEMENTARY MATERIAL}

See supplementary material for the isobaric heat capacity, the thermal expansion as well as calculations of the oxygen ion diffusivity for Y-doped cubic $\mathrm{HfO}_{x}$, and a test of additional $\mathrm{EPP}$, the COMB potentials.

\section{ACKNOWLEDGMENTS}

The authors gratefully acknowledge the computing time granted by the John von Neumann Institute for Computing (NIC) and provided on the supercomputer JURECA at Jülich Supercomputing Centre (JSC). This work was supported by the German Science Foundation (DFG) within the framework of SFB 917, Nanoswitches.

${ }^{1}$ V. A. Gritsenko, T. V. Perevalov, and D. R. Islamov, "Electronic properties of hafnium oxide: A contribution from defects and traps," Phys. Rep. 613, $1-20$ (2016).

${ }^{2}$ J. D. Schieltz, J. W. Patterson, and D. R. Wilder, "Electrolytic behavior of yttria-stabilized hafnia," J. Electrochem. Soc. 118, 1257-1261 (1971).

${ }^{3}$ N. Capron, P. Broqvist, and A. Pasquarello, "Migration of oxygen vacancy in $\mathrm{HfO}_{2}$ and across the $\mathrm{HfO}_{2} / \mathrm{SiO}_{2}$ interface: A first-principles investigation," Appl. Phys. Lett. 91, 192905 (2007).

${ }^{4}$ M. Vos, P. L. Grande, D. K. Venkatachalam, S. K. Nandi, and R. G. Elliman, "Oxygen self-diffusion in $\mathrm{HfO}_{2}$ studied by electron spectroscopy," Phys. Rev. Lett. 112, 175901 (2014).

${ }^{5}$ D. Duncan, B. Magyari-Koepe, and Y. Nishi, "Filament-induced anisotropic oxygen vacancy diffusion and charge trapping effects in hafnium oxide RRAM," IEEE Electron Device Lett. 37, 400-403 (2016).

${ }^{6} \mathrm{G}$. Broglia, G. Ori, L. Larcher, and M. Montorsi, "Molecular dynamics simulation of amorphous $\mathrm{HfO}_{2}$ for resistive RAM applications," Modell. Simul. Mater. Sci. Eng. 22, 065006 (2014).

${ }^{7}$ S. Clima, Y. Y. Chen, R. Degraeve, M. Mees, K. Sankaran, B. Govoreanu, M. Jurczak, S. D. Gendt, and G. Pourtois, "First-principles simulation of oxygen diffusion in $\mathrm{HfO}_{x}$ : Role in the resistive switching mechanism," Appl. Phys. Lett. 100, 133102-1-133102-4 (2012).

${ }^{8}$ S. Clima, B. Govoreanu, M. Jurczak, and G. Pourtois, " $\mathrm{HfO}_{x}$ as RRAM material-First principles insights on the working principles," Microelectron. Eng. 120, 13-18 (2014).

${ }^{9}$ S. Clima, Y. Chen, A. Fantini, L. Goux, R. Degraeve, B. Govoreanu, G. Pourtois, and M. Jurczak, "Intrinsic tailing of resistive states distributions in amorphous $\mathrm{HfO}_{x}$ and $\mathrm{TaO}_{x}$ based resistive random access memories," IEEE Electron Device Lett. 36, 769-771 (2015).

${ }^{10}$ L. Skuja, M. Hirano, H. Hosono, and K. Kajihara, "Defects in oxide glasses," Phys. Status Solidi (C) 2, 15-24 (2005).

${ }^{11} \mathrm{~J}$. Swenson and L. Boerjesson, "Correlation between free volume and ionic conductivity in fast ion conducting glasses," Phys. Rev. Lett. 77, 3569-3572 (1996).

${ }^{12} \mathrm{~S}$. Adams and J. Swenson, "Determining ionic conductivity from structural models of fast ionic conductors," Phys. Rev. Lett. 84, 4144-4147 (2000).

${ }^{13}$ A. N. Cormack, J. Du, and T. R. Zeitler, "Alkali ion migration mechanisms in silicate glasses probed by molecular dynamics simulations," Phys. Chem. Chem. Phys. 4, 3193-3197 (2002).

${ }^{14} \mathrm{H}$. Lammert, M. Kunow, and A. Heuer, "Complete identification of alkali sites in ion conducting lithium silicate glasses: A computer study of ion dynamics," Phys. Rev. Lett. 90, 215901 (2003).

${ }^{15}$ C. T. Imrie, I. Konidakis, and M. D. Ingram, "What variable-pressure variable-temperature measurements are telling us about ion transport in glass," Dalton Trans. 2004, 3067-3070.

${ }^{16}$ J. C. Dyre, P. Maass, B. Roling, and D. L. Sidebottom, "Fundamental questions relating to ion conduction in disordered solids," Rep. Prog. Phys. 72, 046501-1-046501-5 (2009).

${ }^{17}$ H. Lammert and A. Heuer, "Simplified interpretation of transport in disordered inorganic ion conductors from vacancy dynamics," Phys. Rev. Lett. 104, 125901 (2010).

${ }^{18}$ G. V. Lewis and C. R. A. Catlow, "Potential models for ionic oxides," J. Phys. C: Solid State Phys. 18, 1149 (1985).

${ }^{19}$ J. Helfferich, F. Ziebert, S. Frey, H. Meyer, J. Farago, A. Blumen, and J. Baschnagel, "Continuous-time random-walk approach to supercooled liquids. I. Different definitions of particle jumps and their consequences," Phys. Rev. E 89, 042603 (2014).

${ }^{20}$ J. H. Harding, "Computer simulation of defects in ionic solids," Rep. Prog. Phys. 53, 1403 (1990).

${ }^{21}$ R. A. De Souza, M. S. Islam, and E. Ivers-Tiffee, "Formation and migration of cation defects in the perovskite oxide $\mathrm{LaMnO}_{3}$," J. Mater. Chem. 9, 1621-1627 (1999).

${ }^{22} \mathrm{M}$. S. Islam, "Ionic transport in $\mathrm{ABO}_{3}$ perovskite oxides: A computer modelling tour," J. Mater. Chem. 10, 1027-1038 (2000). 
${ }^{23}$ G. Balducci, M. S. Islam, J. Kapar, P. Fornasiero, and M. Graziani, "Bulk reduction and oxygen migration in the ceria-based oxides," Chem. Mater. 12, 677-681 (2000).

${ }^{24}$ M. Kilo, C. Argirusis, G. Borchardt, and R. A. Jackson, "Oxygen diffusion in yttria stabilised zirconia-experimental results and molecular dynamics calculations," Phys. Chem. Chem. Phys. 5, 2219-2224 (2003).

${ }^{25}$ J. E. Jaffe, R. A. Bachorz, and M. Gutowski, "Low-temperature polymorphs of $\mathrm{HfO}_{x}$ : A density-functional theory study," Phys. Rev. B 72, 144107 (2005).

${ }^{26}$ J. D. Gale and A. L. Rohl, "The general utility lattice program (GULP)," Mol. Simul. 29, 291-341 (2003).

${ }^{27}$ A. Pedone, G. Malavasi, M. C. Menziani, A. N. Cormack, and U. Segre, "A new self-consistent empirical interatomic potential model for oxides, silicates, and silica-based glasses," J. Phys. Chem. B 110, 11780-11795 (2006).

${ }^{28}$ T.-R. Shan, B. D. Devine, T. W. Kemper, S. B. Sinnott, and S. R. Phillpot, "Charge-optimized many-body potential for the hafnium/hafnium oxide system," Phys. Rev. B 81, 125328 (2010).

${ }^{29}$ W. C. Swope, H. C. Andersen, P. H. Berens, and K. R. Wilson, "A computer simulation method for the calculation of equilibrium constants for the formation of physical clusters of molecules: Application to small water clusters," J. Chem. Phys. 76, 637-649 (1982).

${ }^{30}$ G. J. Martyna, M. E. Tuckerman, D. J. Tobias, and M. L. Klein, "Explicit reversible integrators for extended systems dynamics," Mol. Phys. 87, 11171157 (1996).

${ }^{31}$ I. T. Todorov, W. Smith, K. Trachenko, and M. T. Dove, "DL_POLY_3: New dimensions in molecular dynamics simulations via massive parallelism," J. Mater. Chem. 16, 1911-1918 (2006).

${ }^{32}$ S. P. Waldow and R. A. De Souza, "Computational study of oxygen diffusion along a[100] dislocations in the perovskite oxide $\mathrm{SrTiO}_{3}$," ACS Appl. Mater. Interfaces 8, 12246-12256 (2016).

${ }^{33}$ J. X. Zheng, G. Ceder, T. Maxisch, W. K. Chim, and W. K. Choi, "Firstprinciples study of native point defects in hafnia and zirconia," Phys. Rev. B 75, 104112 (2007).

${ }^{34}$ T. Zacherle, A. Schriever, R. A. De Souza, and M. Martin, “A $b$ initio analysis of the defect structure of ceria," Phys. Rev. B 87, 134104 (2013).

${ }^{35} \mathrm{~K}$. Ando and Y. Oishi, "Diffusion characteristics of actinide oxides," J. Nucl. Sci. Technol. 20, 973-982 (1983).

${ }^{36} \mathrm{~S}$. Beschnitt, T. Zacherle, and R. A. De Souza, "Computational study of cation diffusion in ceria," J. Phys. Chem. C 119, 27307-27315 (2015).
${ }^{37}$ R. J. Packer and S. J. Skinner, "Remarkable oxide ion conductivity observed at low temperatures in a complex superstructured oxide," Adv. Mater. 22, 1613 (2010)

${ }^{38}$ J. W. Fergus, "Electrolytes for solid oxide fuel cells," J. Power Sources 162, 30-40 (2006).

${ }^{39}$ J. B. Goodenough, “Oxide-ion electrolytes,” Annu. Rev. Mater. Res. 33, 91-128 (2003).

${ }^{40}$ S. Zafar, H. Jagannathan, L. F. Edge, and D. Gupta, "Measurement of oxygen diffusion in nanometer scale $\mathrm{HfO}_{2}$ gate dielectric films," Appl. Phys. Lett. 98, 152903 (2011).

${ }^{41}$ M. F. Trubelja and V. S. Stubican, "Ionic conductivity of the fluorite-type hafnia- $\mathrm{R}_{2} \mathrm{O}_{3}$ solid solutions," J. Am. Ceram. Soc. 74, 2489-2494 (1991).

${ }^{42}$ J. Jiang and J. L. Hertz, "On the variability of reported ionic conductivity in nanoscale YSZ thin films," J. Electroceram. 32, 37-46 (2014).

${ }^{43}$ D. Ceresoli and D. Vanderbilt, "Structural and dielectric properties of amorphous $\mathrm{ZrO}_{2}$ and $\mathrm{HfO}_{2}$," Phys. Rev. B 74, 125108 (2006).

${ }^{44}$ C. A. Angell, K. L. Ngai, G. B. McKenna, P. F. McMillan, and S. W. Martin, "Relaxation in glassforming liquids and amorphous solids," J. Appl. Phys. 88, 3113-3157 (2000).

${ }^{45}$ J. Daru and A. Stirling, "Divided saddle theory: A new idea for rate constant calculation," J. Chem. Theory Comput. 10, 1121-1127 (2014).

${ }^{46}$ S. L. Dole, O. Hunter, and C. J. Wooge, "Elastic properties of monoclinic hafnium oxide at room temperature," J. Am. Ceram. Soc. 60, 488-490 (1977).

${ }^{47}$ M. A. Caravaca, J. C. Mio, V. J. Prez, R. A. Casali, and C. A. Ponce, "A $b$ initio study of the elastic properties of single and polycrystal $\mathrm{TiO}_{2}$, $\mathrm{ZrO}_{2}$ and $\mathrm{HfO}_{2}$ in the cotunnite structure," J. Phys.: Condens. Matter 21, 015501 (2009).

${ }^{48}$ A. Sharma, M. Varshney, H. Shin, K. Chae, and S. O. Won, "XANES, EXAFS and photoluminescence investigations on the amorphous Eu: $\mathrm{HfO}_{2}$," Spectrochim. Acta, Part A 173, 549-555 (2017).

${ }^{49}$ A. R. Genreith-Schriever, P. Hebbeker, J. Hinterberg, T. Zacherle, and R. A. De Souza, "Understanding oxygen-vacancy migration in the fluorite oxide $\mathrm{CeO}_{2}$ : An ab initio study of impurity-anion migration," J. Phys. Chem. C 119, 28269-28275 (2015).

${ }^{50}$ T. B. Schrøder and J. C. Dyre, "Computer simulations of the random barrier model," Phys. Chem. Chem. Phys. 4, 3173-3178 (2002).

${ }^{51}$ R. Metzler, J. Jeon, A. G. Cherstvy, and E. Barkai, "Anomalous diffusion models and their properties: Non-stationarity, non-ergodicity, and ageing at the centenary of single particle tracking," Phys. Chem. Chem. Phys. 16, 24128-24164 (2014). 